\title{
The Nomological Net of Knowledge, Self-Reported Knowledge, and Overclaiming in Children
}

Goecke, B. ${ }^{1}$, Schroeders, U. ${ }^{2}$, Zettler, I. ${ }^{3}$, Schipolowski, S. ${ }^{4}$, Golle, J. ${ }^{5}$, and Wilhelm, O. ${ }^{1}$

${ }^{1}$ Ulm University, Ulm, Germany

${ }^{2}$ University of Kassel, Germany

${ }^{3}$ University of Copenhagen, Denmark

${ }^{4}$ Institute for Educational Quality Improvement (IQB), Berlin, Germany

${ }^{5}$ University of Tübingen, Germany

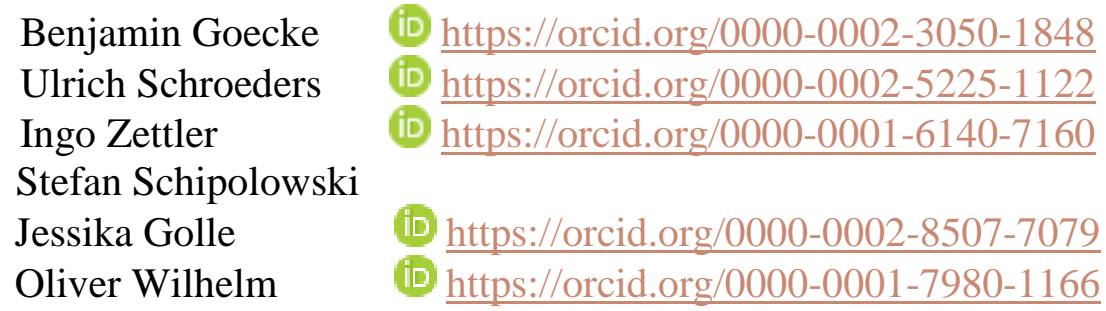

Author Note

The authors have no conflicts of interest to disclose.

Correspondence concerning this article should be addressed to Benjamin Goecke, Institute for Psychology and Pedagogy, Ulm University, Albert-Einstein-Allee 47, 89081

Ulm, Germany. Telephone: +49-(0)731/50 31146. Fax: +49-(0)731/50 31149. E-mail: 
This manuscript is a preprint version of a manuscript that has been accepted for publication in the Journal of Personality Assessment (i.e., after peer-review, but before typesetting).

Update: 04-11-2022

(C) This manuscript is made available under the CC-BY 4.0 license. 
The Nomological Net of Knowledge, Self-Reported Knowledge, and Overclaiming in

\title{
Children
}

\begin{abstract}
Research on self-reported knowledge and overclaiming in children is sparse. With the current study, we aim to close this gap by developing an overclaiming questionnaire measuring selfreported knowledge and overclaiming that is tailored to children. Moreover, we examine the nomological net of self-reported knowledge and overclaiming in childhood discussing three perspectives: Overclaiming as a) a result of deliberate self-enhancement tendencies, b) a proxy for declarative knowledge, and c) an indicator of creative engagement. We juxtaposed overclaiming, as indicated by claiming familiarity with non-existent terms, and self-reported knowledge with fluid and crystallized intelligence, creativity, and personality traits in a sample of 897 children attending third grade. The results of several latent variable analyses were similar to findings known from adult samples: We found no strong evidence for any of the competing perspectives on overclaiming. Just like in adults, individual differences in selfreported knowledge were strongly inflated by overclaiming, and only weakly related to declarative knowledge.
\end{abstract}

Keywords: overclaiming, self-reported knowledge, intelligence, children, epistemic curiosity 
The Nomological Net of Knowledge, Self-Reported Knowledge, and Overclaiming in

\section{Children}

\section{Introduction}

In many clinical and other both applied and basic research settings, people are asked to selfreport their knowledge in certain domains. Exaggerations in measures of self-reported knowledge are also known as overclaiming, a behavioral tendency often measured with overclaiming questionnaires (OCQ; e.g., Paulhus et al., 2003). Overclaiming (OC) is understood as claiming familiarity with terms that do not exist (i.e., foils), whereas selfreported knowledge (SRK) is claiming familiarity with existing terms (i.e., reals). The concept of OC was introduced almost 100 years ago (Raubenheimer, 1925), had a revival in consumer research (Phillips \& Clancy, 1972), and has recently received stronger interest, yielding in a surge of measurement instruments (e.g., Atir et al., 2015; Dunlop et al., 2017, 2019; Goecke et al., 2020; Hülür et al., 2011; Müller \& Moshagen, 2018; Ziegler et al., 2013). In contrast to research with adults, experiences with OC and SRK in children is sparse (for an exception, see, e.g., Butler \& Nelson, 2021).

Although OCQs were successfully applied in PISA 2012 (a worldwide large-scale assessment of scholastic performances of students in schools; OECD, 2013) with adolescents (e.g., Muszyński, 2020), little is known about this behavior in children. This is somewhat surprising given the fact that the initial study, which laid the foundation for today's OCQs, was conducted in a sample of 10-year-old boys (Raubenheimer, 1925) and was set out to quantify their delinquency. Relatedly, a comprehensive overview of potential explanations for OC in children is still missing, whereas the reasons for OC in adults are discussed more frequently (Dunlop et al., 2019; Goecke et al., 2020; Müller \& Moshagen, 2018b). We aim to close this gap and explore the prevalence of OC in children by introducing and psychometrically examining an OCQ for children. Specifically, we investigate which personality and cognitive ability constructs contribute to individual differences in OC and 
SRK in children, including openness to experience, honesty-humility, epistemic curiosity, fluid and crystallized intelligence, and divergent thinking.

\section{Scoring of OCQs}

Research related to OC is shaped by the use of conventional OCQs, containing both reals and foils (e.g., Paulhus et al., 2003); alternative approaches (e.g., Ackerman \& Ellingsen, 2014) are less popular. The prevailing scoring approach for OCQs is based on signal-detection analysis. In this approach, familiarity ratings on reals and foils are translated into dichotomous variables which built the basis for computing $d^{\prime}$, also called $O C$ accuracy, and $c$, also called $O C$ bias. These OC technique indices supposedly distinguish a person's sensitivity to accurately categorize reals as existing and foils as non-existing (OC accuracy), and a tendency to claim familiarity with both reals and foils (OC bias; Paulhus, 2011). Both indices were proposed to quantify the tendency to accurately report one's own knowledge and to overclaim in both item types. Although the basic idea might be appealing, utility of both indices for individual differences research has been questioned due to statistical shortcomings (i.e., they are computationally dependent on each other) and their inconclusiveness regarding the underlying cognitive processes (i.e., lower scores in OC bias can be achieved by a better hit rate, by a lower false alarm rate, or both; see Goecke et al., 2020). This notwithstanding, we appreciate the traditional application of this approach in the context of OCQs and thus provide a set of results and a description of the corresponding formulas regarding signal detection indices in the supplementary materials (see SM Figure 1 and SM Table 4).

A more parsimonious and straightforward scoring approach of OCQs uses familiarity ratings on reals and foils as indicators of two experimentally and computationally distinct psychological entities: SRK and OC. SRK is probed by letting participants rate their familiarity with reals and OC is indicated by claiming familiarity with foils (Hülür et al., 2011).

\section{What Causes OC Behavior in Children?}


To the best of our knowledge, there is one recent study examining OC in children (Butler \& Nelson, 2020). In this study, OC as conceptualized within the signal-detection framework was compared between children and adults by administering a child-friendly OCQ to both groups. Prior to comparing both age groups the convergent validity between the childfriendly OCQ and the OCQ-150 (Paulhus et al., 2003) was investigated in the same sample of adults. Convergent validity, based on correlations between adults' false alarm rates and accuracies (i.e., OC accuracy) only amounted to $r=.51$, and $r=.39$, respectively. Based on the group comparison in the child-friendly OCQ the study concluded that children overclaim more than adults presumably due to different levels of domain-specific knowledge (sensu Ackerman \& Ellingsen, 2014). Butler and Nelson (2020) suggest that developmental trajectories in OC should be considered in future research. We suggest caution in generalizing these results, predominantly due to the limited convergent validity of the child-friendly OCQ with the OCQ-150 and the limited evidence concerning invariance of the scale across age.

Several perspectives on what drives OC behavior (in adults) have been proposed. First, OC was interpreted as being driven by self-enhancement (e.g., Paulhus et al., 2003), which might be, among others, related to the personality trait honesty-humility (Goecke et al., 2020). Second, OC accuracy has been described as a proxy for cognitive abilities (Paulhus \& Harms, 2004), that is, a useful indicator of a person's knowledge. Third, OC was discussed as some kind of creative engagement (Dunlop et al., 2017) and creative investment traits like openness to experience (Hülür et al., 2011). Although these perspectives are derived by research on OC in adults, they should be a helpful guide for investigating OC in children.

According to the first perspective, people engage in OC because they want to present themselves in a desirable way. One reason for this behavior could be that being (or pretending to be) knowledgeable is socially valued. Connecting OC with a general tendency and proneness to self-enhance is not that simple, however. Theories on socially desirable responding (e.g., Paulhus, 2002) distinguish between intentional and unintentional response 
behaviors, but typical OCQs cannot evaluate underlying intentions. OC could be seen as driven by self-deceptive (i.e. unintentional) enhancement tendencies (Paulhus \& Reid, 1991), but was also proposed to be efficient measuring the systematic (i.e., intentional) tendency to misrepresent oneself (Paulhus et al., 2003; Paulhus \& Harms, 2004). The distinction might depend on test settings (e.g., Dunlop et al., 2019). Although the evidence for people showing OC is less convincing in low-stakes than in high-stakes settings (Dunlop et al., 2019), OC seems to be related with narcissism in both (high-stakes: Bensch et al., 2019; low-stakes: Grosz et al., 2017; Ludeke \& Makransky, 2016).

Inferring from this view, that is, OC grasps some sort of intentional or unintentional socially desirable responding behavior, OC might be correlated with honesty-humility. This makes sense for two reasons. First, there is a link between aversive personality traits like narcissism and OC, and honesty-humility has been shown to be the opposite pole of a higherorder factor explaining such aversive traits like narcissism (Schreiber \& Marcus, 2020). Second, dishonesty explains individual differences in impression management (de Vries et al., 2014), which in turn correlate with OC (Bensch et al., 2017). Whereas OC supposedly captures exaggeration of what a person knows, low honesty-humility should reflect deceptive or dishonest characteristics more broadly. Although theoretically plausible, the empirical link between OC and honesty-humility is weak in adults (Dunlop et al., 2019; Goecke et al., 2020; Steger et al., 2020). Importantly, though, this association has not been tested in children, so far. If an association between honesty-humility and OC in children was found, this would indicate a change of the association across age and could therefore be taken as an indicator that the reasons for engaging in OC change over the course of development.

Regarding the second perspective, OC and SRK have also been related to fluid (Gf) and crystallized (Gc) intelligence, because SRK and OC accuracy have been proposed as proxies of cognitive abilities (Paulhus \& Dubois, 2014; Paulhus \& Harms, 2004). It was argued that, because the OC accuracy index is thought to capture a person's sensitivity to 
accurately categorize reals as existing and foils as non-exisiting, it should be associated with higher levels of knowledge, or (crystallized) cognitive abilities in general (Paulhus \& Harms, 2004). This notion remained largely unsupported, however: Correlations between Gc and SRK, or between Gc and OC accuracy usually did not exceed $r=.50-$ too little to justify using SRK as a sufficient proxy for Gc. In addition, SRK is inflated by OC, another reason why the hypothesis found little support in the literature (Goecke et al., 2020; Hülür et al., 2011). These incongruencies between self-reported and objectively measured abilities in adults are in line with meta-analyses (Freund \& Kasten, 2012) and might also affect children. In fact, children have been reported to be not fully capable of giving reliable self-estimates about their skills and abilities (Schneider, 2008). Children are overconfident and overestimate their own performances across a wide range of contexts (e.g., cognitive tasks, Finn \& Metcalfe, 2014) - effects that supposedly also apply to self-reported knowledge.

Predicting OC and SRK with cognitive abilities (i.e., Gf and Gc) might further our understanding about OC and SRK in children and thus provides an insight into the developmental aspects of OC behavior. For example, the magnitude of the relationship between Gc and SRK could inform us about the amount of congruency between questionnaire and test data of the same underlying latent variable. In this vein, it might also be interesting to study gender differences of SRK and OC. More specifically, a majority of studies using latent variable approaches for depicting Gc indicate a male advantage in children aged 5 to 16 years (Härnqvist, 1997; Keith et al., 2008; Reynolds et al., 2008, 2013). The fact that adolescent boys were also found to be more likely than girls to engage in OC (Vonkova et al., 2018) begs the question whether these mean differences can still be observed in children after differences in Gc have been accounted for.

The third perspective is that OC might be considered a form of creative engagement, because rating familiarity with non-existing terms could be indicative of rather uncritical thinking style towards new things. This might be shown in relations with creativity (Goecke et 
al., 2020), but also in associations with broader creative investment traits such as epistemic curiosity (EC) or openness to experience (Bensch et al., 2017; Dunlop et al., 2017, 2019; Goecke et al., 2020). Although creativity, as measured by divergent thinking, is not related to OC in adults (Goecke et al., 2020), this association has never been examined in children. EC was identified as overlapping with openness to experience and intellect (Mussel, 2013), and is conceptualized as the desire to obtain new knowledge either producing positive experiences of intellectual interest or of reducing undesirable conditions of informational deprivation (Piotrowski et al., 2014). EC motivates knowledge acquisition and exploratory behaviour (Berlyne, 1954), and therefore individual differences in this trait might also be associated with OC and SRK. Children high in EC interest might be prone to rate being familiar with foils, because they do not realize the non-existence of items and are generally fascinated by "new" concepts, which could eventually lead to accepting such terms more easily. In other words, OC behavior could partly indicate curiousness. EC deprivation, by contrast, refers to the idea that one is discouraged because one does not fully understand a concept (Litman, 2008). Consequently, children might erroneously claim familiarity with foils, because they are proactively working against that frustration by claiming familiarity with reals and foils to reduce their uncertainty (i.e., deprivation EC might be associated with OC).

In addition to divergent thinking as an ability measure of creative engagement and epistemic curiosity, OC should be correlated with the broader personality trait of openness to experience. People with high levels in this trait may be more prone to overclaim as they are more willing to encounter, engage in, and elaborate on new and unfamiliar ideas - in this vein, OC could be understood as being particularly uncritical towards terms one has never heard of. The empirical results paint an inconclusive picture regarding this association, however. In fact, correlations between openness to experience and OC are moderate at best (e.g., Dunlop et al., 2017). 


\section{Purpose of the Current Study}

Research regarding OC and self-reported knowledge in children is still sparse. We know that children engage in OC, but our understanding about factors possibly driving OC is limited. In addition, little is known about the amount of OC in children, if there are differences between boys and girls, or how reliable OC can be measured in children at all. With the exception for a recent study on OC in children (Butler \& Nelson, 2020), there are no published OCQs for children. Additionally, studying OC in children might help to further our limited understanding about the construct. If one of the perspectives regarding OC finds a more pronounced relation between OC and one of the juxtaposed constructs in children in comparison to adults, this would indicate developmental trajectories of this behaviour which have been neglected so far. and could then be tested more thoroughly. One purpose of our study is thus to replicate previous findings in a new hitherto understudied sample, namely children.

Our research aims are thus as follows: First, we investigate the psychometric properties of a newly developed child-OCQ with 104 items in a large sample of $3^{\text {rd }}$ graders by fitting a confirmatory factor model, which is analogous to previous measurement models for adults (Goecke et al., 2020; Hülür et al., 2011). We expect that SRK and OC are highly related to each other. Second, we investigate gender differences in OC and SRK by means of multigroup confirmatory factor analysis. Adolescent boys have been found to be more likely to engage in OC (Vonkova et al., 2018), which is why we expect to find slightly more OC in boys than in girls, even after differences in knowledge have been accounted for. Third, we juxtapose both OC and SRK with declarative knowledge and fluid intelligence, divergent thinking, honesty-humility, openness to experience, and epistemic curiosity to describe their location within a nomological net. The research objectives and hypotheses were not preregistered. 


\section{Participants and Procedure}

The data for the current study were collected as part of a larger educational program, the Hector Children's Academy Program (for details see Golle et al., 2018), from a subsample of $N=897$ children $(M=8.59, S D=0.47$ years; $47.4 \%$ female $)$ of complete regular school classes. A booklet design with planned missing data was applied for the study, which is why not all scales were administered to the full sample. Each of 71 classes was tested on two subsequent days and there was a total of 17 booklets, which were randomly assigned to the classes. A professional contractor, independent of the research team, was responsible for the data collection. Students were not rewarded or graded in any way and parents gave informed consent prior to testing. The study was voluntary for all participants.

\section{Measures}

\section{Overclaiming Questionnaire}

OC and SRK were assessed with a new set of 104 items, containing 86 reals and 18 foils, resembling typical real-to-foil ratios of other OCQs (e.g., Goecke et al., 2020; Paulhus et al., 2003). Items were tailored to the typical knowledge of third graders as indicated in school curricula and can be assigned to one of 17 knowledge areas covering three broad knowledge domains (nature and technology, art and culture, politics and society). The OCQ was administered in two pseudo-parallel forms, only differing in the sequence of items. The response scale was fully labeled and similar to formats that have been successfully used in other studies (e.g., Goecke et al., 2020; Hülür et al., 2011). Participating children were instructed to indicate their familiarity to all presented terms on a 5-point scale $(1=$ Never heard of it before, $2=$ Heard of it before, but don't know anything about it, $3=I$ know a little about it, $4=I$ know a fair amount about this, and $5=I$ know all about this) within 18 minutes. The presence of foils was not instructed. The OCQ was completed by $N=897$ children; each item was on average answered by $97.8 \%$ of the sample. We present the complete German 
questionnaire and descriptive statistics for all items in the supplementary materials (SM Table $1)$.

\section{Fluid and Crystallized Intelligence}

Students' fluid and crystallized intelligence was measured with an age-adapted version of the BEFKI (Berlin Test of Fluid and Crystallized Intelligence; see e.g., Schroeders et al., 2020), suited for primary school children (Schroeders et al., 2016). The Gf test was comprised of nine verbal, nine numerical, and 16 figural reasoning items and was administered in two parallel forms. Gc was measured with two parallel test forms compiled of 42 knowledge items per version across three broad content domains (humanities, social sciences, and natural sciences). Gf and Gc were completed by $N=848$ participants.

\section{Divergent Thinking}

A verbal divergent thinking test (Carroll, 1993) based on Guilford's Alternative Uses Task (Guilford, 1967) was used. The test consisted of two items requiring students to name within 2 min as many ideas as possible about what could be done with a wooden board and a brick, respectively. Answers were counted by three raters after being checked for meaningfulness and duplicates, thus tapping the fluency aspect of divergent thinking (Weiss et al., 2020). $N=205$ children completed this task.

\section{Personality}

Personality was measured as other-report, completed by one or both of the children's parents. We administered a newly developed and age-adapted other-report, based on the HEXACO Model of Personality (Ashton \& Lee, 2007; Zettler et al., 2020), covering the personality factors of Honesty-Humility, Emotionality, Extraversion, Agreeableness, Conscientiousness, and Openness to Experience with 10 items each. Parents indicated their agreement with the corresponding statements on a five-point scale ranging from "strongly disagree" to "strongly agree". The other report was filled out for $N=562$ children by one 
parent, and sometimes by both parents per child. In the latter case, parents agreed upon their responses before answering the questionnaire.

\section{Epistemic Curiosity}

We assessed epistemic curiosity with a newly developed and age-appropriate scale containing 14 items. In line with scales measuring epistemic curiosity in adults, each item was supposed to tap either interest or deprivation (Litman, 2008; Litman \& Mussel, 2013), resulting in 7 items per dimension. Whereas interest refers to curiosity that is associated with joy regarding new information, deprivation refers to the idea that one is more concerned with decreasing precariousness. Items were statements (e.g., "I am pleased to learn something new.") and children had to indicate how often these statements applied to them on a 4-point scale $(1=$ never, $2=$ seldom, $3=$ often, $4=$ always $)$. The epistemic curiosity questionnaire was completed by $N=192$ children.

\section{Statistical Analyses}

All analyses were conducted with R (R Core Team, 2020) in the framework of Structural equation modeling (SEM) with the R package lavaan (Rosseel, 2012). To reduce model complexity, we used item parcels as indicators after establishing the unidimensionality of the scales (Little et al., 2002). More detailed descriptions of the parcels, the unidimensional measurement models, reliabilities per scale, as well as information regarding the preparation of the data and the computation of parcels can be found in the supplementary materials. We modeled OC and SRK as latent variables with parcels of foils and reals, respectively, as indicators. The six parcels per scale were built randomly, but in a way that each parcel was stratified across all broad content domains (Cole et al., 2016). Based on these parcels, we tested for possible effects of the nested data structure (children nested in classes) but found on average only $2.7 \%$ of variance in these indicators explained by the class-level, whereas the rest of the variance was accounted for by the individual-level. We thus ignored clustering in the subsequent analyses (Pornprasertmanit et al., 2014). Where possible, we used full 
information maximum likelihood estimation under the assumption that missing information was missing completely at random to combine missing data handling and parameter estimation in a single step (Schafer \& Graham, 2002). Models based on dichotomous indicators are based on the Weighted Least Squares Means and Variance adjusted (WLSMV) estimator (Beauducel \& Herzberg, 2006); in this case, pairwise observations were used. Models with continuous indicators are based on a maximum likelihood estimator with robust standard errors (MLR). Good model fit was indicated by CFI (Comparative Fit Index) $\geq .95$, RMSEA (Root Mean Square Error of Approximation) $\leq .06$, and the SRMR (Standardized Root Mean Square Residual) $\leq .08$ (Hu \& Bentler, 1999).

Gender differences in OC and SRK when controlling for Gc levels were studied in a multigroup confirmatory factor analysis (MGCFA) using a reference group approach with boys being the reference group (Schroeders \& Gnambs, 2020). We tested necessary measurement invariance conditions with progressively restrictive stages (Vandenberg \& Lance, 2000). First, we conducted a configural invariance test to establish a baseline model across groups. Second, we tested for metric invariance and constrained factor loadings to equality across groups. Third, we conducted a scalar invariance model, in which the factor loadings and the intercepts were constrained to equality across groups. This type of invariance is the precondition for comparing latent group means. The standardized latent mean of the comparison group can be interpreted as Cohen's $d$ (Cohen, 1988), given that the reference group identification approach has been used. Lastly, we checked for strict measurement invariance, by adding restrictions on the residuals across the groups. Invariance tests were examined by assessing changes in the CFI with values >.01 (Chen, 2007) as indication of substantial model deterioration.

To make all analyses reproducible, we provide all materials necessary, including a complete correlation matrix with descriptive statistics for all variables, and data along with the syntax for the statistical analysis in an online repository: https://osf.io/94bk8/ 


\section{Results}

We report means and standard deviations of mean familiarity ratings of reals and foils comprising the broad knowledge domains of the OCQ (Table 1). Descriptive statistics are comparable to those found in adult samples, that is, the range of the statistics of the parcels is similar to values of other OCQs used in the literature (Dunlop et al., 2019; Goecke et al., 2020; Hülür et al., 2011). Please note that the kurtosis of the foils was lighter tailed in our study, however, indicating somewhat more positive familiarity ratings (OC) of the sample.

\section{Table 1}

Descriptive Statistics for Familiarity Ratings, Including the Means, Standard Deviations, Skewness, and Kurtosis for Each OCQ Parcel.

\begin{tabular}{ccccccccc}
\hline & & & \multicolumn{3}{c}{ Reals } & \multicolumn{3}{c}{ Foils } \\
\cline { 3 - 8 } Parcel & $\begin{array}{c}n \\
\text { (reals })\end{array}$ & $\begin{array}{c}n \\
\text { (foils })\end{array}$ & $M(S D)$ & Skewness & Kurtosis & $M(S D)$ & Skewness & Kurtosis \\
\hline 1 & 15 & 3 & $2.97(0.85)$ & 0.33 & -0.46 & $2.00(1.16)$ & 1.10 & 0.20 \\
2 & 15 & 3 & $3.00(0.81)$ & 0.30 & -0.29 & $1.76(1.03)$ & 1.47 & 1.33 \\
3 & 14 & 3 & $2.96(0.89)$ & 0.29 & -0.69 & $1.79(1.09)$ & 1.50 & 1.39 \\
4 & 14 & 3 & $2.49(0.84)$ & 0.92 & 0.47 & $1.67(1.02)$ & 1.72 & 2.23 \\
5 & 14 & 3 & $3.18(0.80)$ & 0.07 & -0.42 & $1.74(1.05)$ & 1.62 & 1.88 \\
6 & 14 & 3 & $2.76(0.87)$ & 0.59 & -0.16 & $1.78(1.05)$ & 1.44 & 1.26 \\
\hline
\end{tabular}

\section{Measurement Models}

Fit indices of unidimensional measurement models based on item parcels for all measures (see Table 2) indicate good model fit, with the exception of the RMSEA for the SRK model (Model 2). The reliability of all latent variables was acceptable. Measurement models for intelligence (Gf and Gc) and epistemic curiosity included a correlation between the two postulated latent factors, which were in the expected range (e.g., Litman, 2008; Schroeders et al., 2016). We do not report fit indices for the measurement model of fluency, because this scale only contained two indicators. 


\section{Table 2}

Model Fit for the Measurement Models of All Scales.

\begin{tabular}{lccccccccccc}
\hline $\begin{array}{c}\text { Measurement } \\
\text { Model }\end{array}$ & $\begin{array}{c}n \\
\text { (Parcels) }\end{array}$ & $\begin{array}{c}n \\
\text { (Persons) }\end{array}$ & $\chi^{2}$ & $d f$ & CFI & RMSEA & {$[90 \%$ CI $]$} & SRMR & $\omega$ & $r(S E)$ \\
\hline 1 & OC & 6 & 897 & 18.20 & 9 & .993 & .034 & {$[.017 ; .050]$} & .010 & .94 & \\
2 & SRK & 6 & 897 & 55.51 & 9 & .992 & .076 & {$[.059 ; .094]$} & .009 & .97 & \\
3 & Gf / Gc & 6 & 848 & 28.63 & 8 & .984 & .055 & {$[.034, .078]$} & .023 & $.69 / .74$ & $.77(.03)$ \\
4 & $\begin{array}{c}\text { EC } \\
\text { Int / Dep }\end{array}$ & 6 & 210 & 13.31 & 8 & .986 & .056 & {$[.000 ; .103]$} & .032 & $.82 / .73$ & $.82(.05)$ \\
5 & $\begin{array}{c}\text { Honesty- } \\
\text { Humility }\end{array}$ & 4 & 578 & 0.47 & 2 & 1.00 & .000 & {$[.000 ; .051]$} & .013 & .81 & \\
6 & $\begin{array}{c}\text { Openness to } \\
\text { Experience }\end{array}$ & 4 & 577 & 5.39 & 2 & .990 & .054 & {$[.000 ; .107]$} & .017 & .73 & \\
\hline
\end{tabular}

Note. EC = Epistemic Curiosity; Int = Interest; Dep = Deprivation. For both Models 3 and 4, two correlated factors were specified. $\omega=$ McDonald's Omega (McDonald, 1999).

A latent regression model, in which SRK is predicted by OC (see Figure 1), was previously established in adult samples (Goecke et al., 2020; Hülür et al., 2011) and is the basis for all following models. OC predicting SRK considers that an individual's SRK might be affected by OC. OC accounted for $62.3 \%$ of the variance in SRK. In comparison, this regression was somewhat weaker in two adult samples where OC accounted for $50.5 \%$ (Goecke et al., 2020) and 43.6\% of SRK (Hülür et al., 2011), respectively. This indicates that SRK in children might be more inflated by OC in comparison to adults.

\section{Figure 1}

Structural Model of OC and SRK 


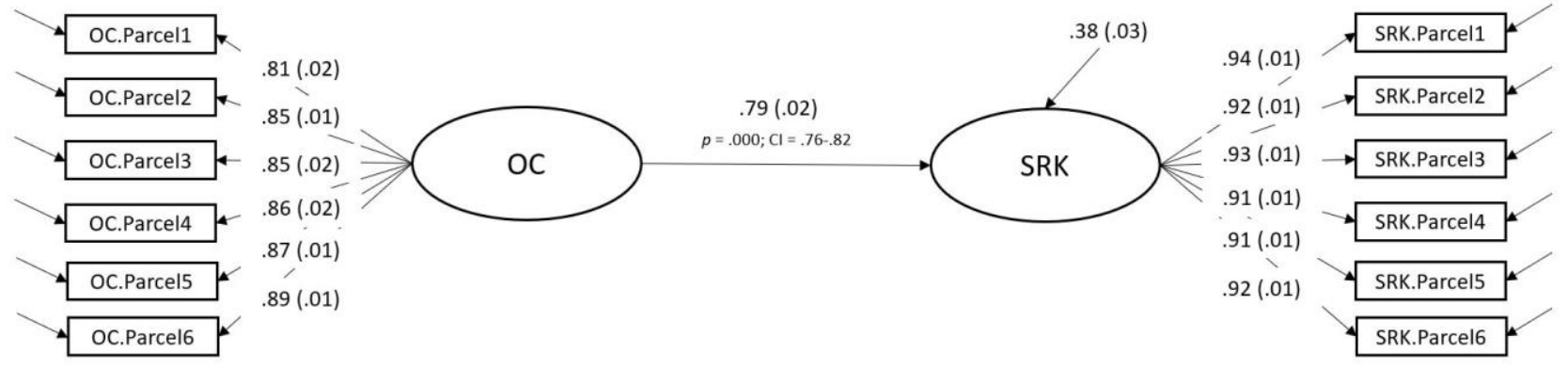

Note. $\mathrm{CI}=95 \%$ Confidence Interval. All parameters are standardized. $n=897 ; \chi^{2}(53)=396.1$, $\mathrm{CFI}=.962, \mathrm{RMSEA}=.085, \mathrm{SRMR}=.040$.

Juxtaposing OC and SRK with the parent-rated personality traits honesty-humility and openness to experience represent the perspective of OC being the result of an intentional strategy to manage others impressions or an open-minded personality (Figure 2). However, OC was neither predicted by openness to experience nor by honesty-humility, whereas SRK was significantly but only infirmly predicted by openness to experience. The non-substantial regression coefficients from honesty-humility and openness to experience to OC is in line with earlier findings with adults (e.g., Dunlop et al.; Goecke et al., 2020), the non-substantial regression weight from openness to experience towards OC adds evidence to earlier, inconsistent findings.

\section{Figure 2}

Structural Model of OC, SRK, Openness to Experience, and Honesty-Humility. 


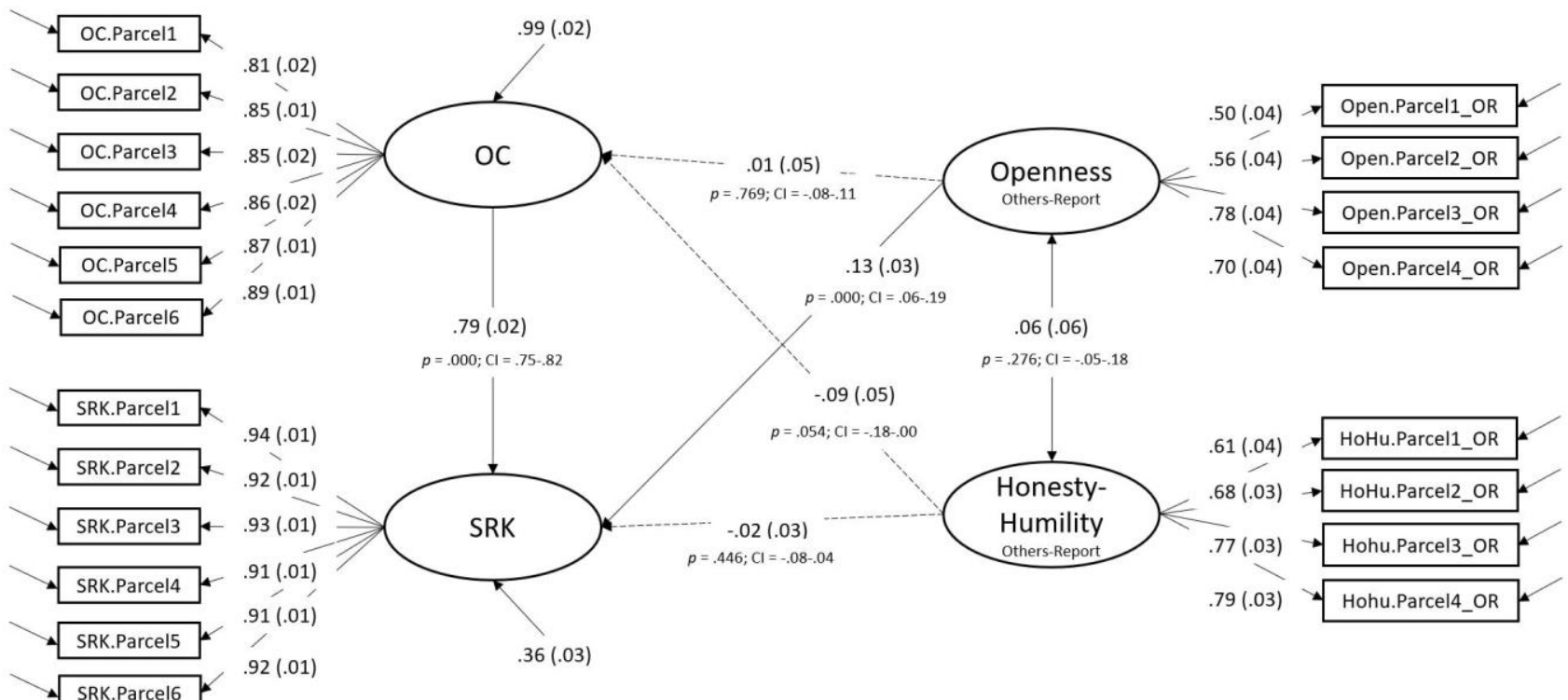

Note. $\mathrm{CI}=95 \%$ Confidence Interval. All parameters are standardized. Non-significant coefficients are displayed with dashed lines $(p>.05) . n=897 ; \chi^{2}(164)=626.8, \mathrm{CFI}=.960$, $\mathrm{RMSEA}=.056, \mathrm{SRMR}=.040$.

Next, we juxtaposed the previously established measurement models of OC and SRK with Gf, Gc, and fluency (see Figure 3). Fluency was modeled together with Gf and Gc, because all three constructs should be understood as abilities that require maximal performance behaviour. Only $8 \%$ of the variance in OC was explained, with small regression coefficients of Gf and fluency towards OC. Gf predicted OC negatively and fluency predicted it positively. Gc did not account for individual differences in OC. These findings slightly deviate from findings in adults, where OC was unrelated to all three constructs (Goecke et al., 2020; Hülür et al., 2011).

SRK was more strongly explained by OC than by actual knowledge. In total, $72.3 \%$ of the variance in SRK was explained by OC and Gc, again resembling findings for adult samples (Goecke et al., 2020; Hülür et al., 2011). The moderate prediction of SRK by Gc is in line with meta-analytical findings regarding the congruency of self-reported and objectively measured abilities (e.g., Freund \& Kasten, 2012). 


\section{Figure 3}

Structural Model of OC, SRK, Gc, Gf, and Fluency.

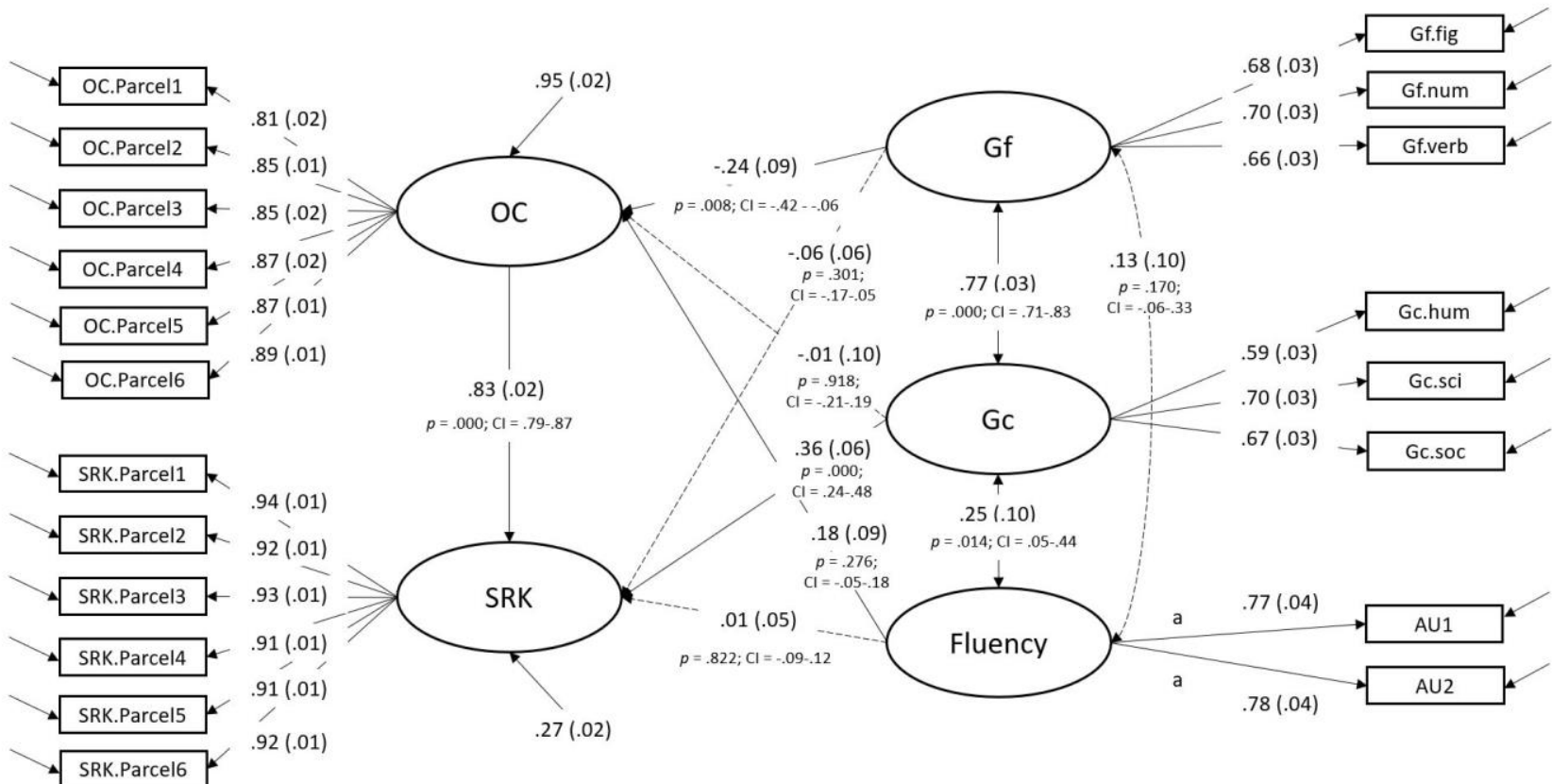

Note. $\mathrm{CI}=95 \%$ Confidence Interval. All parameters are standardized. Non-significant coefficients are displayed with dashed lines $(p>.05) . n=897 ; \chi^{2}(161)=652.7, \mathrm{CFI}=.961$, $\mathrm{RMSEA}=.058, \mathrm{SRMR}=.041$.

Latent mean differences between boys $(n=447)$ and girls $(n=423)$ in OC and SRK were studied by first fitting a slightly adjusted model in which OC and SRK were predicted by Gc, and in which the residuals of both latent factors were correlated. Because a strict measurement invariant model holds (see SM Table 3 in the supplementary materials), means of all factors can be interpreted. As we used boys as the reference group, the latent mean parameter estimates of the girls depict the gender differences (i.e., standardized mean differences; see parameter estimates in Table 3). Looking at the results, girls had lower means on all factors with small effect sizes (see the $d$ estimates in Table 3). In line with the literature, boys showed slightly more actual knowledge (Gc) than girls (Keith et al., 2008). However, boys also tend to overclaim more knowledge than girls, as depicted in higher scores on OC 
and SRK (see also Vonkova et al., 2018). The relations between the three postulated factors were by and large very similar across groups, indicating that the self-reported knowledge scores of girls are as inflated by overclaiming as the boys' scores, even when controlling for Gc.

Table 3

Parameter Estimates of Strict Invariance Model Depicting OC, SRK, and Gc.

\begin{tabular}{|c|c|c|c|c|}
\hline & $\begin{array}{l}\text { Standardized Mean } \\
\text { Differences }(d)\end{array}$ & $95 \%-\mathrm{CI}$ & & \\
\hline SRK & -0.26 & {$[-0.41 ;-0.10]$} & & \\
\hline OC & -0.28 & {$[-0.45 ;-0.11]$} & & \\
\hline \multirow[t]{3}{*}{$\mathrm{Gc}$} & -0.21 & {$[-0.37 ;-0.05]$} & & \\
\hline & Standardized Estim & & & \\
\hline & Boys & $95 \%-\mathrm{CI}$ & Girls & $95 \%-\mathrm{CI}$ \\
\hline$r(\mathrm{SRK}, \mathrm{OC})$ & .86 & {$[.82 ; .90]$} & .82 & {$[.78 ; .86]$} \\
\hline$\beta(\mathrm{OC} \sim \mathrm{Gc})$ & -.17 & {$[-.28 ;-.06]$} & -.19 & {$[-.30 ;-.07]$} \\
\hline$\beta(\mathrm{SRK} \sim \mathrm{Gc})$ & .21 & {$[.10 ; .32]$} & .15 & {$[.03 ; .26]$} \\
\hline
\end{tabular}

Note. $d=$ Cohen's $d$. The standardized mean differences depict the standardized means of the

girls (boys as reference). Negative values of $d$ indicate lower means for girls, whereas positive values of $d$ indicate higher means for girls.

Finally, OC and SRK were regressed onto epistemic curiosity (see Figure 4). Both factors of epistemic curiosity did not account for substantial shares of variance in OC and SRK.

\section{Figure 4}

Structural Model of OC, SRK, and Epistemic Curiosity. 


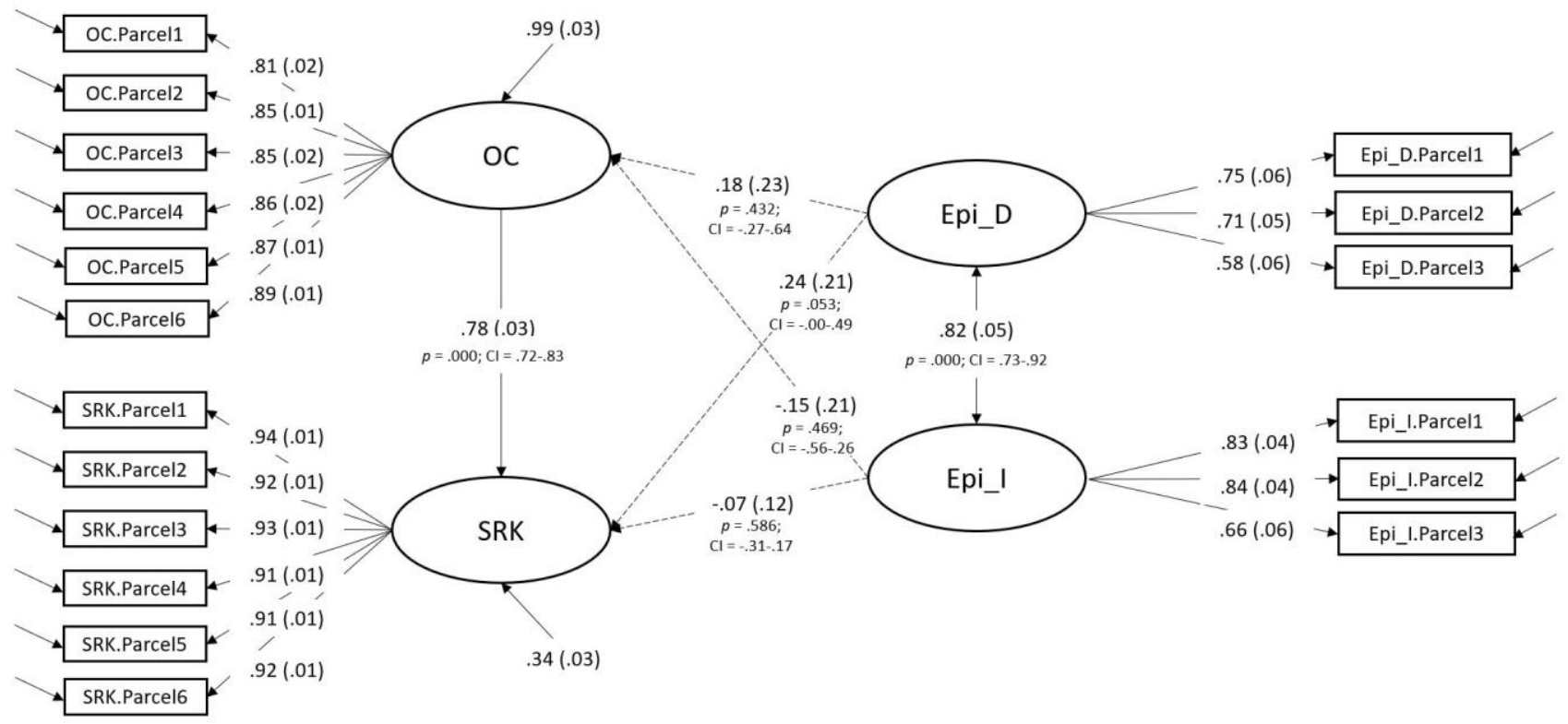

Note. Epi_D = Epistemic Curiosity Deprivation; Epi_I = Epistemic Curiosity Interest; $\mathrm{CI}=$ 95\% Confidence Interval. Parameters are standardized; non-significant coefficients are displayed with dashed lines $(p>.05) . n=897 ; \chi^{2}(153)=559.6, \mathrm{CFI}=.961, \mathrm{RMSEA}=.061$, $\mathrm{SRMR}=.042$.

\section{Discussion}

The literature concerning OC and SRK in adults is broad and so are the corresponding interpretations of this behavior. OC has been mostly studied in normal populations, but is has also been linked to possibly maladaptive personality traits like narcissism (e.g., Grosz et al., 2017) or problematic self-enhancement tendencies (e.g., Paulhus et al., 2003) that might distort self-report questionnaires. Surprisingly, little is known about this behavior in children, even though the construct originated in child development research, where it was introduced as a measure of deviant behaviour of boys (Raubenheimer, 1925).

The aims of the present study were (a) to develop an OCQ for children and to evaluate its psychometric properties, and (b) to study gender differences in OC and SRK, and place both constructs into a broad nomological net. We thus developed an OCQ for children tapping 17 specific knowledge areas from three broad knowledge domains. We embedded both OC and SRK in the nomological net of maximal cognitive performance (Gc, Gf, and fluency) and 
typical behavior (honesty-humility, openness to experience, and epistemic curiosity). In the next sections, we summarize and discuss our findings and sketch their implications.

\section{Perspectives on Overclaiming}

The pattern of results support that we developed a psychometrically sound OCQ. We used reals and foils to distinguish between OC and SRK. This is a straightforward approach to score an OCQ. The range of the descriptive statistics for all items and parcels were comparable to those of OCQs administered in adult samples (e.g., Goecke et al., 2020; Hülür et al., 2011). The newly developed child-OCQ exhibits the same structure as OCQs for adults (e.g., Goecke et al., 2020; Hülür et al., 2011), namely, that responses on the OCQ can be modelled with two factors, where one factor captures the communality of the foils and the other factor captures the communality of the reals. Moreover, SRK of children was considerably affected by OC, with slightly more variance explained in SRK than usually found in adults. This should be taken as evidence that children already show OC (see also Butler \& Nelson, 2020) and confirms studies showing that children tend to overestimate their abilities (e.g., Finn \& Metacalfe, 2014; Schneider, 2008). In summary, it is possible to measure OC and SRK with a conventional OCQ in children. We tested competing perspectives on why persons engage in OC (Goecke et al., 2020) by putting the most prevalent interpretations of OC in adults to a test in children. The overall aim was to lay the foundation for a better understanding of OC by relating the results found in adults to the ones found in children.

A first perspective predicts that OC should be related to honesty-humility, if persons intentionally claim knowledge they cannot have (Paulhus, 1984). Comparable to results from OC in adults (e.g, Dunlop et al., 2019; Goecke et al., 2020; Müller \& Moshagen, 2018a; Steger et al., 2020), this was not the case in our study with children. OC was unrelated with honesty-humility, suggesting that dishonest children typically do not engage more or less in OC than others. Although small but substantial relations between OC and more aversive 
personality traits like narcissism exist, the lack of an association between OC and honestyhumility suggests that OC occurs unintentionally in children. It has to be noted, however, that honesty-humility rather reflects preferences for interpersonal behavior and less intentions to present oneself in a better light, which is why the perspective of OC being reflective of intentional tendencies to misrepresent oneself should not be disregarded in the future.

We also found no support for the second perspective, which connects the responses on OCQs with cognitive abilities - particularly Gc (Paulhus \& Dubois, 2014; Paulhus \& Harms, 2004). OC was unrelated to Gc and only weakly predicted by Gf. SRK was more inflated by OC than it was predicted by Gc. The magnitude of the relation between SRK and Gc underlines that SRK cannot serve as a valid proxy for actual knowledge. Gf and fluency were essentially unrelated with SRK. Thus, we argue that self-reports of cognitive abilities in children cannot serve as a valid measure of these constructs, similar to adult samples although some overlap between these constructs exist (e.g., Freund \& Kasten, 2012). SRK is better understood as a misjudgment and misperception regarding familiarity with existing terms instead. Gender differences in OC and SRK after controlling for individual differences in Gc showed that boys tended to overclaim more and also indicated more SRK (e.g., Vonkova et al., 2018). When considering the Gc levels, however, it becomes apparent that the amount of variance in SRK explained by OC is similar for both genders. Thus, both girls and boys overestimate their knowledge when rating familiarity with reals.

Lastly, we examined the perspective that $\mathrm{OC}$ is associated with forms of creative engagement and creative investment traits such as openness to experience or EC (e.g., Dunlop et al., 2017; Goecke et al., 2020; Hülür et al., 2011). This perspective takes OC as indicative of an open-minded manner to be familiar with unfamiliar terms (i.e., predominantly foils), referring to the idea that new but yet unknown terms (such as foils) might be "enriched" by subjects scoring higher in openness to experience, epistemic curiosity, or creativity, due to their inherent willingness to find meaning in new things. Importantly, though, this tendency 
could also be seen as an uncritical adoption of concepts that do not exist. We tested this idea by regressing both OC and SRK onto openness to experience and epistemic curiosity on the personality side, and onto fluency on the maximal cognitive performance side. By and large, our results add to the conclusion that this perspective lacks support: Openness to experience was not predictive of OC, and only weakly predicted SRK. Both facets of epistemic curiosity, interest and deprivation, explained no variance in OC and SRK. In contrast to an adult sample (Goecke et al., 2020), fluency as an indicator of creativity was not predictive of SRK, but was able to explain a small amount of variance in OC. In total, we found no support for this interpretation of OC.

Apart from the interpretations of OC taken above, we argue that at least some mechanisms underlying OC in children should be attributed to their developing metacognition (Schneider, 2008). Children with highly developed metacognitive knowledge can be considered experts regarding their own knowledge - they know if they know something or not - which is readily related to OC. In the same vein, it was argued that overclaiming should be connected to metacognitive knowledge (Clariana et al., 2016), because OCQs tap into the so-called feeling of knowing (Paulhus, 2011), which is considered a part of metacognitive knowledge. In theory, this is the part of metacognitive knowledge that helps people decide if they know or do not know a specific information. This is exactly what is required of participants when they have to fill out an OCQ. They see terms and have to decide whether they are familiar with them or not. In children, declarative metacognitive knowledge increases with age and is associated with age-related improvements in cognitive and school performances (Schneider \& Löffler, 2016). In other words, the developments of cognitive abilities and metacognitive knowledge seem to be intertwined. Thus, future studies examining OC in children should consider a metacognitive perspective on OC.

\section{On the Use of OCQs}


In the present study, we showed that overclaiming expressed as rating the familiarity with foils can be reliably measured in children and is strongly related with self-reported knowledge. With respect to the validity of OC, we replicated earlier critical results established in adult samples. The nomological net of OC, as we tested it, provides insufficient support for the different perspectives on OC that have been proposed in the literature. In turn, one might argue that one should not only conceptualize OC as measured by conventional OCQs as a valuable construct of individual differences since the evidence so far regarding its predictive validity for real-world outcomes is rather stuttering (Watts et al., 2021), although some studies give reason to be optimistic (e.g., Bing et al., 2011; Dunlop et al., 2016). In order to make sure that this conclusion is not premature, studies that systematically vary test design or juxtapose the OCQ with other measures of OC might be warranted. In the following, we present a number of related research questions that are to our knowledge largely unanswered.

Research related to OC is shaped by the use of conventional setups of OCQs, that is, it is characterized by a number of default settings that might or might not be optimal in a psychometric sense. This is reflected in a large number of different OCQs described in the literature (e.g., Atir et al., 2015; Butler \& Nelson, 2021; Dunlop et al., 2017, 2019; Goecke et al., 2020; Hülür et al., 2011; Mesmer-Magnus et al., 2006; Paulhus et al., 2003; Ziegler et al., 2013). These OCQs usually comprise $80 \%$ reals and 20\% foils (Paulhus, 2011), and items are restricted to a number of knowledge domains (e.g., Paulhus et al., 2003). The fixed ratio of reals and foils might be seen as an unnecessary restriction that rests on the presupposition that other ratios will not change the rank order of participants. Other things being equal, a more conservative approach of expressing familiarity will presumably be beneficial if the base rate of foils is high. Moreover, high conceptual proximity, relative plausibility, or only orthographical resemblances of foils to existing concepts is likely to deceive otherwise knowledgeable participants into endorsing familiarity with foils. The question how foils should be constituted is not conclusively answered. In addition to that, most OCQs are 
administered without informing participants about the presence of foils and their base rate (e.g., Paulhus et al., 2003). Knowledgeable and intelligent participants might infer the presence of foils, specifically in domains in which they have expertise. This inference partly removes blindness of participants towards their treatment and participants that infer the presence of foils are likely to adjust their response behavior. Taken together, systematically varying the base rate of foils and manipulating the information about their presence in the instructions should help us understand to what extent results found in studies with OCQs are generalizable.

More general, the method of OCQs should be scrutinized critically. The basic idea of rating familiarities with reals and foils is hardly different from conventional vocabulary tests, although conventional vocabulary tests show considerably more convergent validity with other measures of Gc (Schipolowski et al., 2014). The traditional OCQ format could be varied to gradually shift OCQs towards vocabulary tests. For instance, the response formats of both tests could be aligned, in order to test the relation of OCQs to more traditional vocabulary tests depending on different response formats: word/non-word decisions, finding synonyms or antonyms, choosing the existing word among non-words, and finally: decide whether one knows a term or not.

For the most part, OCQs have been applied in the context of traditional knowledge domains, often by inquiring about the familiarity with single words. The rational of the technique also allows studying familiarity with factitious or fictitious statements and measures could also include less traditional domains such as familiarity with dance moves, novel music, rumors about celebrities etc. So far, we have limited knowledge whether or not the technique is more useful in these less academic contexts.

There are also a number of methodological challenges of the prevalent signal detection approach (Paulhus, 2011) that have not been addressed adequately yet. For example, there is no conventional measurement model. The coherence of both reals and foils is neglected. The 
relation between reals and foils and how and why this relation might vary on an item level is also neglected although this covariance is clearly essential for the traditional indices derived in OCQs (i.e., the hit rate, the false-alarm rate, and eventually $d$ ' and $c$ ). These indices, which are ratio variables, are logically and computationally dependent (Zimprich et al., 2020). These issues deserve more attention if the signal detection approach is meant to continue to be the method of choice for scoring OCQs.

In summary, the utility of the OCQ-technique strikes us as limited so far. The suggestions presented in this section might help to endorse a more final verdict, one way or another.

\section{Where Do We Go From Here?}

The previous literature on $\mathrm{OC}$ seems to be consensual about the fact that we can reliably observe individual differences in OC as measured with OCQs across numerous contexts (i.e., low- and high-stakes settings). The construct of OC has gained considerable attention in the last 10 years - not to mention its' application in PISA 2012. Unfortunately, however, individual differences in OC are not stably associated with other constructs of interest — both personality traits and cognitive abilities — across various studies. This raises the question of what OC actually reflects. Empirically supported answers to this question should be conceived as a presupposition for using OC in applied contexts. Large multivariate studies measuring OC might help us to better understand the very nature of this behavior, but we argue that these studies should not be limited to a single operationalization of OC.

In fact, research on a construct should usually transcend the methods of inquiry. OC should go beyond what a test presumably measures - i.e. exaggerating one's knowledge by falsely claiming familiarity with a word. Other operationalizations of OC are thinkable and desirable. If the purpose of a measurement instrument is to detect exaggerations regarding a person's knowledge, then OC could also be measured, for example, by letting participants first answer knowledge questions by stating whether or not they know the correct response 
and have them at a later stage answer the questions by retrieving or recognizing the correct response. Another method could be to ask participants to respond to fictional knowledge questions in an open-ended response format, for example, "Which researcher won the Nobel prize for physics in 1700?". Participants who do not stat "I don't know" or "There is no answer to this question" are then engaging in OC. Relatedly, the combination between actual knowledge tests and self-ratings regarding one's own knowledge could also initiate research towards a possible counterpart of OC; underclaiming, or belittling oneself. It seems fruitful to vary the response format of instruments measuring $\mathrm{OC}$ to further the understanding of the construct. Thus, it would be possible to disentangle the measurement from the construct. From an overarching perspective, engaging in self-serving and exaggerating behavior also comprises tweaking biographical events, malingering symptoms for gaining monetary benefits, presenting character strengths in speed-dating, and the like. The broadness of this domain is not reflected in available measurement instruments for OC, however, although OC might be relevant behavior in these contexts. In light of our current results and the previous literature on OC, we thus argue that a multi-method approach should be warranted for furthering our understanding about overclaiming as a construct of individual differences. Such an approach might also inform us about the utility of measuring $\mathrm{OC}$ for diagnostic purposes in clinical and other applied settings, like high-stake application contexts where identifying distorted self-reports and individuals engaging in faking might be helpful.

\section{Conclusion}

The present study contributes to the hitherto sparse literature on OC and SRK in children. Children seem to overclaim their knowledge at least as much as adults do, but the predictors of this behavior are unknown in both children and adults. OC should be considered a reliable latent variable of individual differences, but it has little to do with honesty-humility, cognitive abilities, or creative investment traits. If OC will be studied in the future, it should 
be investigated with a more systematic variation of the stimulus material and with multiple methods - in both children and adults. 


\section{Funding}

The work reported herein was supported by grants from the Hector Foundation II.

\section{Author Note}

The authors have no conflicts of interest to disclose.

\section{Data Accessibility Statement}

The study materials, data, and analysis scripts used for this article can be accessed at https://osf.io/94bk8/ 


\section{References}

Ackerman, P. L., \& Ellingsen, V. J. (2014). Vocabulary overclaiming - A complete approach: Ability, personality, self-concept correlates, and gender differences. Intelligence, 46, 216-227. https://doi.org/10.1016/j.intell.2014.07.003

Ashton, M. C., \& Lee, K. (2007). Empirical, Theoretical, and Practical Advantages of the HEXACO Model of Personality Structure. Personality and Social Psychology Review, 11(2), 150-166. https://doi.org/10.1177/1088868306294907

Atir, S., Rosenzweig, E., \& Dunning, D. (2015). When knowledge knows no bounds: Selfperceived expertise predicts claims of impossible knowledge. Psychological Science, 26(8), 1295-1303. https://doi.org/10.1177/0956797615588195

Beauducel, A., \& Herzberg, P. Y. (2006). On the Performance of Maximum Likelihood Versus Means and Variance Adjusted Weighted Least Squares Estimation in CFA. Structural Equation Modeling: A Multidisciplinary Journal, 13(2), 186-203. https://doi.org/10.1207/s15328007sem1302_2

Bensch, D., Maaß, U., Greiff, S., Horstmann, K. T., \& Ziegler, M. (2019). The nature of faking: A homogeneous and predictable construct? Psychological Assessment, 31(4), 532-544. https://doi.org/10.1037/pas0000619

Bensch, D., Paulhus, D. L., Stankov, L., \& Ziegler, M. (2017). Teasing Apart Overclaiming, Overconfidence, and Socially Desirable Responding. Assessment, 351-363. https://doi.org/10.1177/1073191117700268

Berlyne, D. E. (1954). A Theory of Human Curiosity. British Journal of Psychology, 43(3), $180-191$.

Bing, M. N., Kluemper, D., Kristl Davison, H., Taylor, S., \& Novicevic, M. (2011). Overclaiming as a measure of faking. Organizational Behavior and Human Decision Processes, 116(1), 148-162. https://doi.org/10.1016/j.obhdp.2011.05.006 
Butler, J. R., \& Nelson, N. L. (2021). Children overclaim more knowledge than adults do, but for different reasons. Journal of Experimental Child Psychology, 201, 104969. https://doi.org/10.1016/j.jecp.2020.104969

Carroll, J. B. (1993). Human cognitive abilities: A survey of factor-analytic studies. Cambridge University Press.

Chen, F. F. (2007). Sensitivity of Goodness of Fit Indexes to Lack of Measurement Invariance. Structural Equation Modeling: A Multidisciplinary Journal, 14(3), 464504. https://doi.org/10.1080/10705510701301834

Clariana, M., Castelló, A., \& Cladellas, R. (2016). Feeling of knowing and over-claiming in students from secondary school to university. Learning and Individual Differences, 49, 421-427. https://doi.org/10.1016/j.lindif.2016.05.008

Cohen, J. (1988). Statistical power analysis for the behavioral sciences (2nd ed). L. Erlbaum Associates.

Cole, D. A., Perkins, C. E., \& Zelkowitz, R. L. (2016). Impact of homogeneous and heterogeneous parceling strategies when latent variables represent multidimensional constructs. Psychological Methods, 21(2), 164-174. https://doi.org/10.1037/met0000047

de Vries, R. E., Zettler, I., \& Hilbig, B. E. (2014). Rethinking Trait Conceptions of Social Desirability Scales: Impression Management as an Expression of Honesty-Humility. Assessment, 21(3), 286-299. https://doi.org/10.1177/1073191113504619

Dunlop, P. D., Bourdage, J. S., de Vries, R. E., Hilbig, B. E., Zettler, I., \& Ludeke, S. G. (2017). Openness to (reporting) experiences that one never had: Overclaiming as an outcome of the knowledge accumulated through a proclivity for cognitive and aesthetic exploration. Journal of Personality and Social Psychology, 113(5), 810-834. https://doi.org/10.1037/pspp0000110 
Dunlop, P. D., Bourdage, J. S., de Vries, R. E., McNeill, I. M., Jorritsma, K., Orchard, M., Austen, T., Baines, T., \& Choe, W.-K. (2019). Liar! Liar! (when stakes are higher): Understanding how the overclaiming technique can be used to measure faking in personnel selection. Journal of Applied Psychology. https://doi.org/10.1037/ap10000463

Finn, B., \& Metcalfe, J. (2014). Overconfidence in children's multi-trial judgments of learning. Learning and Instruction, 32, 1-9. https://doi.org/10.1016/j.learninstruc.2014.01.001

Freund, P. A., \& Kasten, N. (2012). How smart do you think you are? A meta-analysis on the validity of self-estimates of cognitive ability. Psychological Bulletin, 138(2), 296-321. https://doi.org/10.1037/a0026556

Goecke, B., Weiss, S., Steger, D., Schroeders, U., \& Wilhelm, O. (2020). Testing competing claims about overclaiming. Intelligence, 81, 101470. https://doi.org/10.1016/j.intell.2020.101470

Golle, J., Zettler, I., Rose, N., Trautwein, U., Hasselhorn, M., \& Nagengast, B. (2018). Effectiveness of a “Grass Roots” Statewide Enrichment Program for Gifted Elementary School Children. Journal of Research on Educational Effectiveness, 11(3), 375-408. https://doi.org/10.1080/19345747.2017.1402396

Grosz, M. P., Lösch, T., \& Back, M. D. (2017). The narcissism-overclaiming link revisited. Journal of Research in Personality, 70, 134-138. https://doi.org/10.1016/j.jrp.2017.05.006

Guilford, J. P. (1967). The nature of human intelligence. McGraw-Hill, Inc.

Härnqvist, K. (1997). Gender and Grade Differences in Latent Ability Variables. Scandinavian Journal of Psychology, 38(1), 55-62. https://doi.org/10.1111/14679450.00009 
Hu, L., \& Bentler, P. M. (1999). Cutoff criteria for fit indexes in covariance structure analysis: Conventional criteria versus new alternatives. Structural Equation Modeling: A Multidisciplinary Journal, 6(1), 1-55. https://doi.org/10.1080/10705519909540118

Hülür, G., Wilhelm, O., \& Schipolowski, S. (2011). Prediction of self-reported knowledge with over-claiming, fluid and crystallized intelligence and typical intellectual engagement. Learning and Individual Differences, 21(6), 742-746. https://doi.org/10.1016/j.lindif.2011.09.006

Keith, T. Z., Reynolds, M. R., Patel, P. G., \& Ridley, K. P. (2008). Sex differences in latent cognitive abilities ages 6 to 59: Evidence from the Woodcock-Johnson III tests of cognitive abilities. Intelligence, 36(6), 502-525. https://doi.org/10.1016/j.intell.2007.11.001

Litman, J. A. (2008). Interest and deprivation factors of epistemic curiosity. Personality and Individual Differences, 44(7), 1585-1595. https://doi.org/10.1016/j.paid.2008.01.014 Litman, J. A., \& Mussel, P. (2013). Validity of the Interest-and Deprivation-Type Epistemic Curiosity Model in Germany. Journal of Individual Differences, 34(2), 59-68. https://doi.org/10.1027/1614-0001/a000100

Little, T. D., Cunningham, W. A., Shahar, G., \& Widaman, K. F. (2002). To Parcel or Not to Parcel: Exploring the Question, Weighing the Merits. Structural Equation Modeling: A Multidisciplinary Journal, 9(2), 151-173. https://doi.org/10.1207/S15328007SEM0902_1

Ludeke, S. G., \& Makransky, G. (2016). Does the Over-Claiming Questionnaire measure overclaiming? Absent convergent validity in a large community sample. Psychological Assessment, 28(6), 765-774. https://doi.org/10.1037/pas0000211

Mesmer-Magnus, J., Viswesvaran, C., Deshpande, S., \& Joseph, J. (2006). Social desirability: The role of over-claiming, self-esteem, and emotional intelligence. Psychological Science, 336-356. 
Müller, S., \& Moshagen, M. (2018a). True Virtue, Self-Presentation, or Both?: A Behavioral Test of Impression Management and Overclaiming. Psychological Assessment, 31(2).

Müller, S., \& Moshagen, M. (2018b). Overclaiming shares processes with the hindsight bias. Personality and Individual Differences, 134, 298-300. https://doi.org/10.1016/j.paid.2018.06.035

Mussel, P. (2013). Intellect: A theoretical framework for personality traits related to intellectual achievements. Journal of Personality and Social Psychology, 104(5), 885906. https://doi.org/10.1037/a0031918

Muszyński, M. (2020). Validity of the overclaiming technique as a method to account for response bias in self-assessment questions. Analysis on the basis of the PISA 2012 data [University at Krakow]. https://ruj.uj.edu.pl/xmlui/bitstream/handle/item/251494/muszynski_validity_of_the_o verclaiming_technique_as_a_method_2020.pdf?sequence=1\&isAllowed=y

Paulhus, D. L. (1984). Two-component models of socially desirable responding. Journal of Personality and Social Psychology, 46(3), 598-609. http://dx.doi.org/10.1037/00223514.46.3.598

Paulhus, D. L. (2002). Socially Desirable Responding: The Evolution of a Construct. In H. I. Braun, D. N. Jackson, \& D. E. Wiley (Eds.), The role of constructs in psychological and educational measurement (pp. 49-69). Erlbaum Associates, Inc.

Paulhus, D. L. (2011). Overclaiming on personality questionnaires. In M. Ziegler, C. MacCann, \& R. D. Roberts (Eds.), New perspectives on faking in personality assessement (pp. 151-164). Oxford University Press, Inc.

Paulhus, D. L., \& Dubois, P. J. (2014). Application of the Overclaiming Technique to Scholastic Assessment. Educational and Psychological Measurement, 74(6), 975-990. https://doi.org/10.1177/0013164414536184 
Paulhus, D. L., \& Harms, P. D. (2004). Measuring cognitive ability with the overclaiming technique. Intelligence, 32(3), 297-314. https://doi.org/10.1016/j.intell.2004.02.001

Paulhus, D. L., Harms, P. D., Bruce, M. N., \& Lysy, D. C. (2003). The over-claiming technique: Measuring self-enhancement independent of ability. Journal of Personality and Social Psychology, 84(4), 890-904. https://doi.org/10.1037/0022-3514.84.4.890

Paulhus, D. L., \& Reid, D. B. (1991). Enhancement and Denial in Socially Desirable Responding. Journal of Personality and Social Psychology, 60(2), 307-317.

Phillips, D. L., \& Clancy, K. J. (1972). Some effects of "social desirability" in survey studies. American Journal of Sociology, 77(5), 921-940. https://doi.org/10.1086/225231

Piotrowski, J. T., Litman, J. A., \& Valkenburg, P. (2014). Measuring Epistemic Curiosity in Young Children: Brief Report. Infant and Child Development, 23(5), 542-553. https://doi.org/10.1002/icd.1847

Pornprasertmanit, S., Lee, J., \& Preacher, K. J. (2014). Ignoring Clustering in Confirmatory Factor Analysis: Some Consequences for Model Fit and Standardized Parameter Estimates. Multivariate Behavioral Research, 49(6), 518-543. https://doi.org/10.1080/00273171.2014.933762

R Core Team. (2020). R: A language and environment for statistical computing. $\mathrm{R}$ Foundation for Statistical Computing. https://www.R-project.org/

Raubenheimer, A. S. (1925). An experimental study of some behavior traits of the potentially delinquent boy. Psychological Monographs, 34, 1-107.

Reynolds, M. R., Keith, T. Z., Flanagan, D. P., \& Alfonso, V. C. (2013). A cross-battery, reference variable, confirmatory factor analytic investigation of the CHC taxonomy. Journal of School Psychology, 51(4), 535-555. https://doi.org/10.1016/j.jsp.2013.02.003

Reynolds, M. R., Keith, T. Z., Ridley, K. P., \& Patel, P. G. (2008). Sex differences in latent general and broad cognitive abilities for children and youth: Evidence from higher- 
order MG-MACS and MIMIC models. Intelligence, 36(3), 236-260. https://doi.org/10.1016/j.intell.2007.06.003

Rosseel, Y. (2012). lavaan: An R Package for Structural Equation Moeling. Journal of Statistical Software, 48, 1-36.

Schafer, J. L., \& Graham, J. W. (2002). Missing data: Our view of the state of the art. Psychological Methods, 7(2), 147-177. https://doi.org/10.1037//1082-989X.7.2.147

Schipolowski, S., Wilhelm, O., \& Schroeders, U. (2014). On the nature of crystallized intelligence: The relationship between verbal ability and factual knowledge. Intelligence, 46, 156-168. https://doi.org/10.1016/j.intell.2014.05.014

Schneider, W. (2008). The Development of Metacognitive Knowledge in Children and Adolescents: Major Trends and Implications for Education. Mind, Brain, and Education, 2(3), 114-121. https://doi.org/10.1111/j.1751-228X.2008.00041.X

Schneider, W., \& Löffler, E. (2016). The Development of Metacognitive Knowledge in Children and Adolescents. In J. Dunlosky \& S. K. Tauber (Eds.), The Oxford Handbook of Metamemory. Oxford University Press.

Schreiber, A., \& Marcus, B. (2020). The place of the "Dark Triad" in general models of personality: Some meta-analytic clarification. Psychological Bulletin, 146(11), 10211041. https://doi.org/10.1037/bu10000299

Schroeders, U., \& Gnambs, T. (2020). Degrees of Freedom in Multigroup Confirmatory Factor Analyses: Are Models of Measurement Invariance Testing Correctly Specified? European Journal of Psychological Assessment, 36(1), 105-113. https://doi.org/10.1027/1015-5759/a000500

Schroeders, U., Schipolowski, S., \& Wilhelm, O. (2020). Berliner Test zur Erfassung fluider und kristalliner Intelligenz für die 5. Bis 7. Klasse (BEFKI 5-7). Hogrefe Verlag. 
Schroeders, U., Schipolowski, S., Zettler, I., Golle, J., \& Wilhelm, O. (2016). Do the smart get smarter? Development of fluid and crystallized intelligence in 3rd grade. Intelligence, 59, 84-95. https://doi.org/10.1016/j.intell.2016.08.003

Steger, D., Schroeders, U., \& Wilhelm, O. (2020). Caught in the act: Predicting cheating in unproctored knowledge assessment. Assessment.

Vandenberg, R. J., \& Lance, C. E. (2000). A Review and Synthesis of the Measurement Invariance Literature: Suggestions, Practices, and Recommendations for Organizational Research. Organizational Research Methods, 3(1), 4-70. https://doi.org/10.1177/109442810031002

Vonkova, H., Papajoanu, O., \& Stipek, J. (2018). Enhancing the Cross-Cultural Comparability of Self-Reports Using the Overclaiming Technique: An Analysis of Accuracy and Exaggeration in 64 Cultures. Journal of Cross-Cultural Psychology, 49(8), 1247-1268. https://doi.org/10.1177/0022022118787042

Watts, L. L., Kuzmich, I., Leung, D. W., Gibson, C., \& Barsa, A. (2021). Impression management and overclaiming on job applications: Related to future sales performance? International Journal of Selection and Assessment, 29(1), 134-138. https://doi.org/10.1111/ijsa.12310

Weiss, S., Steger, D., Kaur, Y., Hildebrandt, A., Schroeders, U., \& Wilhelm, O. (2020). On the Trail of Creativity: Dimensionality of Divergent Thinking and Its Relation With Cognitive Abilities, Personality, and Insight. 22.

Zettler, I., Thielmann, I., Hilbig, B. E., \& Moshagen, M. (2020). The Nomological Net of the HEXACO Model of Personality: A Large-Scale Meta-Analytic Investigation. Perspectives on Psychological Science, 15(3), 723-760. https://doi.org/10.1177/1745691619895036 
Ziegler, M., Kemper, C., \& Rammstedt, B. (2013). The Vocabulary and Overclaiming Test (VOC-T). Journal of Individual Differences, 34(1), 32-40. https://doi.org/10.1027/1614-0001/a000093

Zimprich, D., Nusser, L., \& Pollatos, O. (2020). Are interoceptive accuracy scores from the heartbeat counting task problematic? A comment on Zamariola et al. (2018). Biological Psychology, 152, 107868. https://doi.org/10.1016/j.biopsycho.2020.107868 